\title{
Surgical Approaches to Large Peripheral Nerve Sheets Tumors
}

\author{
Hedaya M. Hendam, Hatem M. El Samouly, Hamdy M. Behairy* \\ Neurosurgery Departments, Faculty of Medicine, Al-Azhar University, Cairo, Egypt \\ Email: *dr.hamdybehairy@hotmail.com
}

How to cite this paper: Hendam, H.M., El Samouly, H.M. and Behairy, H.M. (2018) Surgical Approaches to Large Peripheral Nerve Sheets Tumors. Open Journal of Modern Neurosurgery, 8, 46-56. https://doi.org/10.4236/ojmn.2018.81003

Received: November 4, 2017

Accepted: December 17, 2017

Published: December 20, 2017

Copyright ( $) 2018$ by authors and Scientific Research Publishing Inc. This work is licensed under the Creative Commons Attribution International License (CC BY 4.0).

http://creativecommons.org/licenses/by/4.0/

(c) (i) Open Access

\begin{abstract}
Peripheral nerve sheath tumors (PNSTs) are not rare tumors. Its sound management is complete surgical excision. The aim of this study was to describe clinical presentation, accuracy of different investigations and ideal surgical approaches in relation to outcomes. The study is a prospective and included 26 surgically treated large PNSTs in 22 patients operated through different surgical approaches according to the site of the tumors. The fellow up period was 6 - 60 months. The mean age was 41.8 years, 9 were males and 13 were females. The common presentations were pain in 12 lesions, swelling in 8 lesions, neural deficits in 4 lesions and positive Tinel's test in 2 lesions. These lesions were excised through 6 surgical approaches. Histopathological examination showed 20 benign and 6 malignant lesions. Five cases of malignant peripheral nerve sheet tumors (MPNSTs) had local recurrent, 4 cases had distant metastasis, 4 cases received chemotherapy and radiotherapy, and 4 cases were died in the postoperative fellow up period. It was concluded that surgical approaches to the PNSTs, depends on their locations. Adequate exposure with minimal dissection minimized the neurological deficit and decreased rate of local recurrence.
\end{abstract}

\section{Keywords}

Peripheral Nerve Sheet Tumors

\section{Introduction}

Malignant peripheral nerve sheet tumors (MPNTs) are kinds of soft tissue sarcomas arising from peripheral nerves or thought to be differentiated from nerve sheath elements including Schwann cell, fibroblasts, perineural cells or pluripotent cells of neural crest origin. MPNSTs are rapidly growing, firm, fixed lesions initially causing either progressive loss of function or severe pain [1]. Magnetic 
resonance imaging (MRI) is usually the modality of choice in preoperative evaluation of peripheral nerve sheet tumors. It provides exceptional contrast of soft-tissue structures, high spatial resolution and multi-planar imaging capability [2]. With the all-important clinical evaluation, imaging permits the clinician to identify the size, shape, location, distribution of masses, displaying its relationship to nearby nerve fascicles, determine growth rates when serial imaging studies are available, suggest the nature of the pathological entity and visualize muscle innervation [3]. Magnetic resonance neurography comprises a series of techniques that further enhance the contrast between peripheral nerves and surrounding tissues [4]. Positron emission tomography is the tool of choice when it comes to observing metabolic changes [5]. Plain film radiography and computed tomography scan is still useful in evaluating patients who are not MR compatible, studying secondary changes in skeletal structures, detecting and characterizing calcification in tumors and evaluating the chest and abdomen for signs of metastasis of MPNTs [6]. Ultrasonography is among the most widely available and least time-consuming imaging techniques available for the examination of suspected nerve masses [7].

\section{Patients and Methods}

The present work aimed to describe the clinical presentations of large PNSTs, review the accuracy of different investigations in the diagnosis, the ideal surgical approaches and exposures of these tumors in relation to the outcomes. This study included 26 surgically treated large PNSTs for 22 patients. Patients were undergoing surgery between 2013 and 2016 at Al-Azhar University Hospitals. Tumors located at the intra- or extra-foraminal spinal nerve root level were included. Tumors of intra-spinal or intracranial origin were excluded in this study. All patients had pre-operative detailed history including risk factors for neurofibromatosis I (NFI), presence of spontaneous local or referred pain and numbness. Clinical evaluation was directed to the presence of skin pigmentation, parathesia, weakness, and palpable masses, with stress on size, site, multiplicity, mobility, localized tenderness, and presence of Tinel' sign. All patients had evaluated with plain X-ray, CT scan, MRI, in examination of those lesions. Radiological finding was reviewed to document each tumor location, size, multiplicity, margins, and relationship to adjacent structures. EMG and nerve conduction velocity were also used in the assessment of all lesions. An open biopsy was performed in all patients presenting with clinical and radiological signs suggestive of malignancy. If a MPNST was confirmed, evaluation for metastatic lesions included lung, and abdominal CT scans and technetium liver, spleen and bone scans were conducted. All tumors were undergoing surgery with the aim of total excision. Some patients had double lesions or triple lesions were undergoing multiple surgeries. The patients were undergoing surgery through different surgical approaches depending on the site, size, and the degree of tumor exposure required. Surgical techniques, extend of surgical resection and status of surgical 
margins and surgical complication were also assessed. The techniques used for surgical excision was through exploration of the proximal, distal poles of the tumor and nerve fascicles. With microsurgical technique, the fascicles were gently dissected free of the tumor in the extra-capsular plane. Care was taken to isolate fascicles enter the substance of the tumor or its capsule. Occasionally, debulking of large tumors in order to simplify subsequent dissection is done if MPNST was confirmed, resection of a malignant tumor varied depending on its origin and location. Total resection was the goal, it was often impossible without producing vascular and functional loss. This procedure included removal of the nerve, adjacent soft tissues, and several centimeters safety margin. Histopathological examination for all patients was studied. The adjunctive treatment was given after histopathological confirmation of MPNSTs. Post-operative radiotherapy and/or chemotherapy were applied. The follow up period was ranged from 6 to 60 months. Assessment of local recurrence and metastasis were documented. The outcome of the patients is evaluated relative to the nature of tumors, extent of surgical excision and adjuvant treatment used. Statistical analysis: Data was analyzed by Microsoft Office XP 2010 (excel) and Statistical Package for Social Science (SPSS) version 10. Parametric data was expressed as mean $\pm \mathrm{SD}$, and non-parametric data was expressed as number and percentage of the total.

\section{Results}

We operated 26 PNSTs in 22 patients, with 19 patients had single lesions, two patients had double lesions, and one patient had triple lesions. Nine were males (41.9\%) and 13 were females (59.1\%), with the age ranged from 4 years to 63 years $(38.23 \pm 14.38$ years). Four patients (18\%) have past history NF 1. All tumors were above $5 \mathrm{~cm}$ in diameter (Table 1 ).

The clinical presentation varied: pain was presented in 12 patients, palpable size swellings with variable degree of tenderness in 8 patients, 4 patients presented with motor weakness, and 2 patients had positive Tinel's test along the the involved nerve. Nine lesions were at the para-spinal region (3 cervical, 3 dorsal, and 3 lumbar) related to posterior spinal nerve root, 7 lesions related to the peripheral nerves ( 3 femoral, one redial, one sciatic, one common peroneal and one anterior tibial nerve), 4 lesions in intrathoracic cavity (one mid-dorsal

Table 1 . The incidence of age, sex, and multiplicity among studied patients.

\begin{tabular}{ccc}
\hline Variables & No=22 & $\%$ \\
\hline Age/years: Mean \pm SD & \multicolumn{2}{c}{$38.22 \pm 14.38$} \\
Range & \multicolumn{2}{c}{$59(4-63)$} \\
Sex: Male & 9 & 40.9 \\
Female & 13 & 59.1 \\
Multiplicity: Single & 19 & 86.4 \\
Multiple & 3 & 13.6 \\
\hline
\end{tabular}


and three apical) related to anterior spinal nerve root, 3 lesions were retroperitoneal related to the lumbar plexus, 3 lesions in the supraclavicular area related to brachial plexus. These lesions were excised surgically through 6 surgical approaches: 9 tumors had approaches through isolated posterior spinal, 7 through direct exposure of the involved peripheral nerve, 3 through anterior cervical approaches, 3 through anterolateral intercostal thoracotomies, 2 through retroperitoneal approaches, 2 through combined approaches, (one case was through posterior cervical with high dorsal thoracotomy and other case was through thoracotomy with retroperitoneal approaches). These approaches were done without operative complications or risks, except 4 cases had profound neurological deficit, and one case had pseudo-meningiocele with brachialgia (Table 2).

Histopathological examinations showed, 6 lesions were malignant PNSTs, and 20 lesions were benign PNSTs which, distributed as follow: 13 lesions neurofi-

Table 2. Showing; incidence of clinical presentation, site, surgical approaches, type of surgery, pathology and recurrence among studied patients.

\begin{tabular}{|c|c|c|}
\hline Variables & $\mathrm{No}=26$ & $\%$ \\
\hline \multicolumn{3}{|l|}{ Clinical presentation: } \\
\hline Local pain & 12 & 46 \\
\hline Swelling & 8 & 31 \\
\hline Motor weakness & 4 & 15.4 \\
\hline Tinel's test & 2 & 7.6 \\
\hline Site: Paraspinal & 9 & 34.6 \\
\hline Peripheral limbs & 7 & 27 \\
\hline Intrathoracic & 4 & 15.4 \\
\hline Retroperitoneal & 3 & 11.6 \\
\hline Supraclavicular & 3 & 11.6 \\
\hline \multicolumn{3}{|l|}{ Surgical approaches: } \\
\hline Posterior spinal & 9 & 34.6 \\
\hline Direct exposure & 7 & 27 \\
\hline Anterior cervical & 3 & 11.6 \\
\hline Thoracotomy & 3 & 11.6 \\
\hline Retro-peritoneal & 2 & 7.6 \\
\hline Combined & 2 & 7.6 \\
\hline Pathology: Benign & 20 & 77 \\
\hline Malignant & 6 & 23 \\
\hline Type of surgery: Total & 19 & 73 \\
\hline Partial & 7 & 27 \\
\hline Recurrence: No & 19 & 73 \\
\hline Yes & 7 & 27 \\
\hline
\end{tabular}


bromas, 4 lesions schwannoma, 2 lesions lipomas and one lesion ganglioneuroma. The Extend of surgical excision was total in 19 lesions and partial in 7 lesions. Local recurrence was observed in 7 lesions in the fellow up period, 2 cases were benign, and 5 cases were malignant (Table 3 ).

Four causes of MPNSTs had distant metastasis in the fellow up period. Four cases of MPNSTs received chemotherapy and radiotherapy. The mean follow up period was, (17.54 \pm 13.45$)$. Four cases of MPNSTs were died in the postoperative fellow up period. The other two cases of MPNSTs are still alive, one case had local recurrence after radio and chemotherapy, underwent lower limb amputation, and the second has no recurrence or metastasis (Tables 4-6).

\section{Case Presentation}

\subsection{Case 1}

Female patient, 35 years old, with history of neurofibromatosis I, presented by slow growing left supraclavicular hard swelling, brachialgia, and left upper limb

Table 3. Showing incidence of metastasis, chemo-radiotherapy, follow up and death among studied patients.

\begin{tabular}{|c|c|c|c|}
\hline \multicolumn{2}{|c|}{ Variables } & \multirow{2}{*}{$\begin{array}{c}\mathrm{No}=22 \\
18\end{array}$} & \multirow{2}{*}{$\begin{array}{c}\% \\
81.8\end{array}$} \\
\hline Metastasis: & No & & \\
\hline & Yes & 4 & 18.2 \\
\hline \multirow{2}{*}{ Chemo-radiotherapy: } & No & 18 & 81.8 \\
\hline & Yes & 4 & 18.2 \\
\hline \multirow{3}{*}{ Follow up: } & $\leq 1$ year & 11 & 50.0 \\
\hline & $>1-2$ years & 9 & 40.9 \\
\hline & $>2$ years & 2 & 9.1 \\
\hline \multirow{2}{*}{ Death } & No & 18 & 81.8 \\
\hline & Yes & 4 & 18.2 \\
\hline
\end{tabular}

Table 4. Distribution of recurrence, metastasis, chemo-radiotherapy and death in relation to the pathology.

\begin{tabular}{|c|c|c|c|c|c|c|c|}
\hline \multirow{2}{*}{ Variables } & & \multicolumn{4}{|c|}{ Pathology } & \multirow{2}{*}{$X^{2}$} & \multirow{2}{*}{$P$ value } \\
\hline & & \multicolumn{2}{|c|}{ Benign } & \multicolumn{2}{|c|}{ Malignant } & & \\
\hline \multirow{2}{*}{ Recurrence } & No & 14 & 87.5 & 1 & 16.7 & & \\
\hline & Yes & 2 & 12.5 & 5 & 83.3 & 10.09 & $0.04^{*}$ \\
\hline \multirow[t]{2}{*}{ Metastasis } & No & 16 & 100.0 & 2 & 33.3 & & \\
\hline & Yes & 0 & 0.0 & 4 & 66.7 & 13.04 & $0.02^{\star}$ \\
\hline \multirow[t]{2}{*}{ Chemo-radiotherapy } & No & 16 & 100.0 & 2 & 33.3 & & \\
\hline & Yes & 0 & 0.0 & 4 & 66.7 & 13.04 & $0.02^{*}$ \\
\hline \multirow[t]{2}{*}{ Death } & No & 16 & 100.0 & 2 & 33.3 & & \\
\hline & Yes & 0 & 0.0 & 4 & 66.7 & 13.04 & $0.02^{\star}$ \\
\hline Total & 16 & 100.0 & 6 & 100.0 & & & \\
\hline
\end{tabular}

*significant at $\mathrm{P}<0.05$ 
Table 5. Distributions of metastasis, and death in relation to chemo-radiotherapy among studied patients.

\begin{tabular}{|c|c|c|c|c|c|c|}
\hline \multirow{3}{*}{$\begin{array}{c}\text { Variables } \\
\text { Metastasis: No }\end{array}$} & \multicolumn{4}{|c|}{ Chemo-radiotherapy } & \multirow{3}{*}{$X^{2}$} & \multirow{3}{*}{$P$ value } \\
\hline & \multicolumn{2}{|c|}{ No } & \multicolumn{2}{|c|}{ Yes } & & \\
\hline & 18 & 100.0 & 0 & 00.0 & & \\
\hline Yes & 0 & 0.0 & 4 & 100.0 & 3.32 & 0.13 \\
\hline Death: No & 16 & 88.9 & 2 & 50.0 & & \\
\hline Yes & 2 & 11.1 & 2 & 50.0 & 3.32 & 0.13 \\
\hline Total & 18 & 100.0 & 4 & 100.0 & & \\
\hline
\end{tabular}

${ }^{*}$ significant at $\mathrm{P}<0.05$

Table 6. Distributions of metastasis, recurrence and death in relation to fellow up among studied patients.

\begin{tabular}{|c|c|c|c|c|c|c|c|c|}
\hline \multirow{2}{*}{$\begin{array}{l}\text { Variables } \\
\text { Metastasis: }\end{array}$} & \multicolumn{6}{|c|}{ Fellow up } & \multirow{2}{*}{$X^{2}$} & \multirow{2}{*}{$\begin{array}{c}\mathrm{P} \\
\text { Value }\end{array}$} \\
\hline & \multicolumn{2}{|c|}{$\leq 1$ year } & \multicolumn{2}{|c|}{$>1$ - 2years } & \multicolumn{2}{|c|}{$>2$ years } & & \\
\hline No & 8 & 72.7 & 8 & 88.9 & 2 & 100.0 & & \\
\hline Yes & 3 & 27.3 & 1 & 11.1 & 0 & 0.0 & 1.35 & 0.50 \\
\hline \multicolumn{9}{|l|}{ Recurrence: } \\
\hline No & 8 & 72.7 & 5 & 55.6 & 2 & 100.0 & & \\
\hline Yes & 3 & 27.3 & 4 & 44.4 & 0 & 0.0 & 1.69 & 0.43 \\
\hline \multicolumn{9}{|l|}{ Death: } \\
\hline No & 8 & 72.7 & 8 & 88.9 & 2 & 100.0 & & \\
\hline Yes & 3 & 27.3 & 1 & 11.1 & 0 & 0.0 & 1.35 & 0.50 \\
\hline Total & 11 & 100.0 & 9 & 100.0 & 2 & 100.0 & & \\
\hline
\end{tabular}

*significant at $\mathrm{P}<0.05$

weakness. She was investigated by plain X-ray and MRI; primary diagnosed as PNST, and was operated through supraclavicular Z shaped incision with total removal and neural tissues preservation. Post-operative histopathology was MPNSTs (Figure 1).

\subsection{Case 2}

Male child, 4 years old, presented by difficult gait, right sciatica, lower limb weakness. He was diagnosed with neoplastic lesion related to the sciatic nerve and operated through direct approach to the sciatic nerve in the thigh, with total excision and preservation of sciatic nerve. Histopathological examination: lipoma (Figure 2).

\subsection{Case 3}

Male patient, 45 years old, presented by low back pain and no radicular pain, 


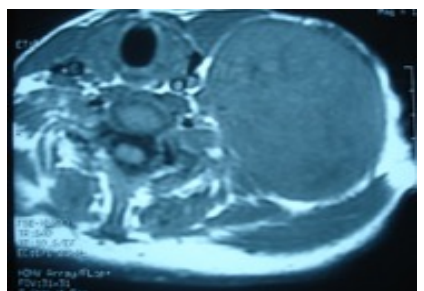

(a)

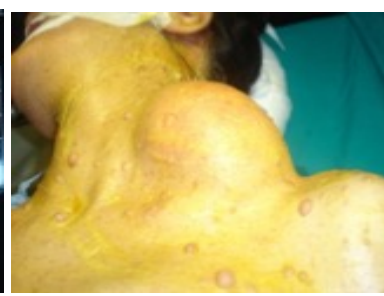

(b)

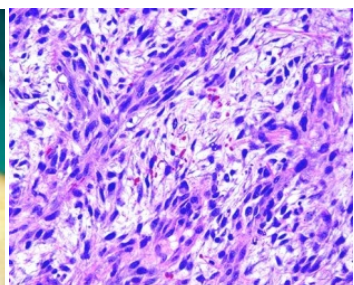

(c)

Figure 1. Showing case 1 presentation. (a) Pre-operative axial MRI of supraclavicular MPNST; (b) pre-operative surgical position; (c) Histopathology, MPNST.

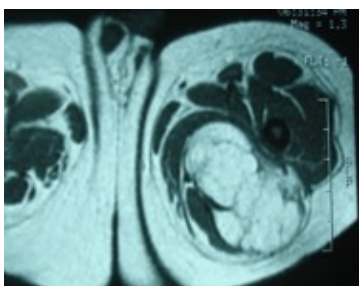

(a)

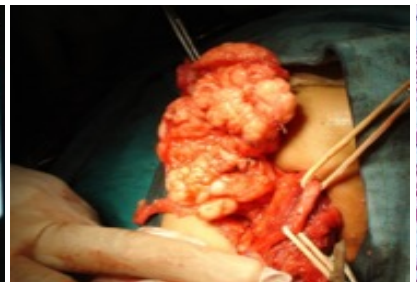

(b)

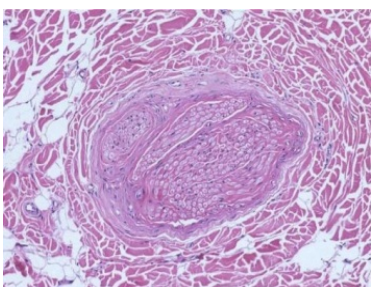

(c)

Figure 2. Showing case 2 presentation. (a) Pre-operative axial MRI of Lt. PNSTs; (b) intraoperative dissection; (c) Histopathology, fibolipomatous hamartoma.

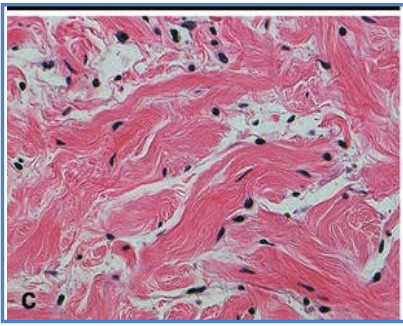

(a)

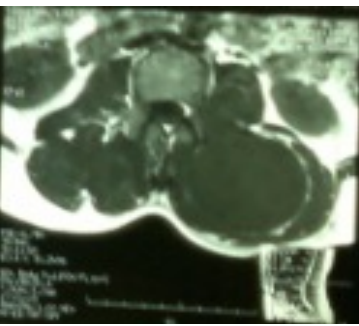

(b)

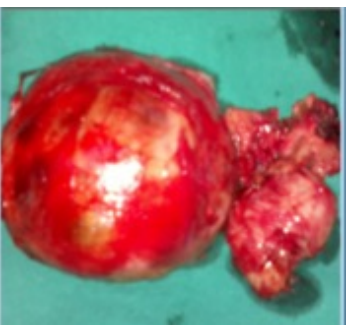

(c)

Figure 3. Showing case 3 presentation. (a) Pre-operative axial T2 MRI with left lumbar paraspinal PNST; (b) Total excision; (c) Histopathology: neurofibroma.

Plain X-ray and MRI finding was left foraminal and paraspinal lesion related to the spinal nerve root. The patient was operated through classical paraspinal approach with total removal. Histopathological examination was neurofibroma (Figure 3).

\section{Discussion}

Many researchers are trying to correlate different features of PNSTs with surgical outcomes, to anticipate which patient will have higher chances of complications. Because of different location, the surgical approach to those tumors is challenger to the surgeon. With the concept the ideal surgical approach is that which allows a good exposure and total excision which relatively reflect on the outcome. The risk factors include genetic factors NFI, radiation and trauma. The most common sites affected are extremities followed by retroperitoneal and trunk [8]. In our study, neurofibromatosis type I was present as risk factor, but we did not report pre-operative radiation or significant trauma with the com- 
mon site in the spine followed by intrathoracic and retroperitoneal in contrast to the previous studies [9]. Among different diagnostic modalities, MRI is generally considered the golden standard; useful to locate the tumor, define its origin, and reveal neurovascular structures. Such information helps proper pre-operative diagnosis to facilitate the safest and most efficacious surgical approach [10]. This is coincident with our review, where MRI was the most efficient method of diagnosis in PNSTs and gives sufficient information to use the proper surgical approach. In our study, review of MRIs revealed no specific feature associated with good surgical outcomes. So, detailed informed consent should be obtained from all patients who proceed to surgery, to ensure that they understand the potential risks of neurological deficit. In our study, the elements of evaluation as accuracy of MRI in diagnosis and lesion characters, tumor's numbers, and type of the lesions were accepted in comparison to study of Knight 2007 as MRI gave 100\% accuracy, there may have been several reasons for this phenomenon. First, his sample size was small (only 20 patients had MRI). Second, his study was neither randomized nor controlled. Nevertheless, MRI is found to be a more accurate modality for diagnosing PNSTs. When the tumor arises from a major nerve trunk, careful planning is important to reduce the risk of neurological damage at operation. The risk is minimized when the diagnosis is made prior to surgery. So, that the clinical team can select a surgeon with appropriate microsurgical expertise and equipment to perform the resection [11]. In our study 14 patients (63.6\%) presented by slowly growing mass using the available radiological methods of diagnosis except positron emission tomography that is not available in our hospital. Also, our treating options were as Longhi et al., 2010, they commonly present with a rapidly enlarging mass [12]. Imaging modalities include computed tomography, magnetic resonance imaging, positron emission tomography scan. Treatment is complete surgical excision with or without radiotherapy, or no adjuvant chemotherapy with surgery [13]. Isolated limb perfusion is used for treating extremity sarcomas for whom amputation is the only option for local treatment [14]. In our study, one patient with MPNST had local recurrence at the thigh and treated through lower limb amputation, after exclusion of other lesions by metastatic work up investigations. Total excision of the lesion is the treatment of choice for PNSTs, as it is well encapsulated and the nerve fiber is displaced, instead of being penetrated. Theoretically, it is possible to remove the lesion without significant nerve deficit. However, even under meticulous surgical dissection and use of microscopic techniques, complications still occur. MPNSTs in our series were 6 patients of little number to give significant statistical data in comparison to others, although we had one 35 years, female patients of 6 MPNSTs associated with NFI, about (16.6\%), in relation to 50\% with Zhu et al., 2012 [1]. This tumor occurs in the age group of 20 to 50 years with an equal male and female predilection [15]. In spite of being uncommon, MPNST have been described in different locations of the body, occasionally associated or not with NFI [10]. Our cases were presented in the extremities, paraspinal region, 
intrathoracic and retroperitoneal space. We did not report other cases in rare location as isolated cases in the parapharyngeal region [16]. The tumors appear as a bosselated, sessile, circumscribed submucosal mass associated with pain or parathesia or muscle weakness and atrophy [16]. This slow enlarging mass exhibits rapid growth and two third of the lesions were more than $5 \mathrm{~cm}$ at the time of diagnosis [11]. In our cases, all lesions were above $5 \mathrm{~cm}$ at time of diagnosis. Despite almost of MPNSTs have an unfavorable outcome to chemotherapy and conventional radiotherapy, there are many studies supported surgical treatment especially by the method of en-bloc excision with negative surgical margin [17]. This was in agreement with our decision in management of MPNSTs, as we operated 6 patients, 4 died during follow up with post-operative chemotherapy and radiotherapy. Recently, a retrospective study revealed that post-operative prognosis for MPNSTs was quite poor with high recurrence and low survival rate, in the condition of positive surgical margin [1]. As demonstrated by previous study that patients with positive surgical margins have 1.8-fold risk of local recurrence and 1.1-fold risk of distant metastasis at 10 years' cumulative incidence [18]. MPNSTs are highly aggressive soft tissue sarcomas that rarely occur sporadically in the general population. However, in patients with NFI, they occur with a lifetime incidence of $8 \%-13 \%$. They account for $5 \%-10 \%$ of soft tissue sarcomas. The incidence of sporadic MPNSTs is low, with a lifetime risk of $0.001 \%$. Most MPNSTs are associated with major nerves of the body wall and extremities. These tumors originate from the nerve sheath rather than from the nerve itself [13]. All ages and both sexes may be affected. Sporadic cases are most common between 40 and 50 years of age while those occurring in the setting of NFI are diagnosed some 10 years earlier. The male and female ratio is 1:1 [9]. The site of MPNSTs, age of the patients and sex distribution in our small number of cases were in acceptance with previous studies. There have been few large studies about survival and those reporting 5-year survival lack consistency, with survival rates in the range of $39 \%-85 \%$. Few studies have suggested NFI as an independent indicator of poor prognosis in MPNSTs [19]. A combination of clinical, pathological, and immunohistochemistry helps in diagnosing of these tumors. Primary site, size, and surgical margins are significant for disease-free survival and overall survival [12]. Korin et al. 2012, reported 5-year overall survival rate of $46 \%$ in patients with MPNSTs [20]. Though multimodality therapy, including surgical resection and adjuvant radiotherapy, the prognosis remains dismal [21]. In our study 2 patients (33\%) out of 6 patients, with MPNSTs, had 5-year free survival after radiotherapy and chemotherapy, approximately similar to the previous studies.

\section{Conclusion}

Peripheral nerve sheath tumors were not rare lesions. There were more benign PNSTs than malignant ones. They presented at any age and sex. Their diagnosis depends on clinical evaluation and radiological examination. MRI was the most 
specific and sensitive in diagnosis. The most common benign tumors were related to the spinal nerve roots and major nerves in the extremities, and were commonly neurofibromas. The malignant PNSTs were related to the sciatic nerve, brachial, pelvic plexus, and spinal nerve roots. The main management of PNSTs was surgical. The surgical approaches depend on the site and size of the tumor that allows a good exposure and minimal dissection. Adjuvant radiotherapy and/or chemotherapy in malignant tumors may be helpful. Total excision achieved by ideal surgical approaches and an experienced surgeon appear to provide the best outcome over all.

\section{References}

[1] Zhu, B., Liu, X., Liu, Z., Yang, S. and Liao, H.I. (2012) Malignant Peripheral Nerve Sheath Tumors of the Spine: Clinical Manifestations, Classification, Treatment, and Prognostic Factors. European Spine Journal, 21, 897-904.

https://doi.org/10.1007/s00586-011-2093-y

[2] Wasa, J., Nishida, Y., Tsukushi, S., Shido, Y., Sugiura, H., Nakashima, H. and Ishiguro, N. (2010) MRI Features in the Differentiation of Malignant Peripheral Nerve Sheath Tumors and Neurofibromas. American Journal of Roentgenology, 194, 1568-1574. https://doi.org/10.2214/AJR.09.2724

[3] Singh, T. and Kliot, M. (2007) Imaging of Peripheral Nerve Tumors. Neurosurgery Focus, 22, E6. https://doi.org/10.3171/foc.2007.22.6.7

[4] Du, R., Auguste, K.I., Chin, C.T., Engstrom, J.W. and Weinstein, P.R. (2010) Magnetic Resonance Neurography for the Evaluation of Peripheral Nerve, Brachial Plexus, and Nerve Root Disorders. Journal of Neurosurgery, 112, 362-371. https://doi.org/10.3171/2009.7.JNS09414

[5] Benz, M.R., Czernin, H., Dry, S.M., Tap, W.D., Allen-Auerbach, M.S., El-ashoff, D., Phelps, M.E., Weber, W.A. and Eilber, F. (2010) Quantitative F18-Fluorodeoxyglucose Positron Emission Tomography Accurately Characterizes Peripheral Nerve Sheath Tumors as Malignant or Benign. Cancer, 116, 451-458. https://doi.org/10.1002/cncr.24755

[6] Sperandio, M, Di Poce, I., Ricci, A., Trapano, R., Costanzo, E., Di Cello, P., Pelle, F. and Izzo, L. (2013) Malignant Peripheral Nerve Sheath Tumor: CT and MRI Findings. Case Reports in Radiology, 51, 78-79.

[7] Lawande, A.D., Warrier, S. and Joshi, M.S. (2014) Role of Ultrasound in Evaluation of Peripheral Nerves. Indian Journal of Radiology and Imaging, 24, 254-258. https://doi.org/10.4103/0971-3026.137037

[8] Nguyen, R.L., Jett, K., Harris, G.J., Cai, W., Friedman, J.M. and Mautner, V.F. (2014) Benign Whole Body Tumor Volume Is a Risk Factor for Malignant Peripheral Nerve Sheath Tumors in Neurofibromatosis Type I. Journal of Neuro-Oncology, 116, 307-313. https://doi.org/10.1007/s11060-013-1293-1

[9] Ziadi, A. and Saliba, I. (2010) Malignant Peripheral Nerve Sheath Tumor of Intracranial Nerve. A Case Series Review. Auris Nasus Larynx, 37, 539-545. https://doi.org/10.1016/j.anl.2010.02.009

[10] Jeong, H.L., Nom, G.K., Kyung, S.L. and Jun, S.K. (2014) Malignant PNSTs in Frontal Sinus, Orbital Cavity and Ethmoid Cavity. Arch Craniofacial Surgery, 15, 125-128. https://doi.org/10.7181/acfs.2014.15.3.125

[11] Chabra, A., Soldatos, T., Durand, D.J., Carrino, J.A., McCarthy, E.F. and Belzberg, A.J. (2011) The Role of Magnetic Resonance Imaging in the Diagnostic Evaluation 
of Malignant Peripheral Nerve Sheath Tumors. Indian Journal of Cancer, 48, 328-334. https://doi.org/10.4103/0019-509X.84945

[12] Longhi, A., Errani, C., Magagnoli, G., Alberghini, M., Gambarotti, M. and Mercuri, M. (2010) High Grade Malignant Peripheral Nerve Sheath Tumors: Outcome of 62 Patients with Localized Disease and Review of the Literature. Journal of Chemotherapy, 22, 413-418. https://doi.org/10.1179/joc.2010.22.6.413

[13] DeVita, V.T., Lawrence, T.S., Rosenberg, S.A., DeVita, H. and Rosenberg C. (2011) Principles and Practice of Oncology. 9th Edition. Wolters Kluwer, Philadelphia, 750-769.

[14] Feig, B.W., Berger, D.H., Fuhrman, G.M. and Anderson, T. (2009) Surgical Oncology Handbook. 4th Edition, Lippincott Williams and Wilkins Publication, Philadelphia, 1245-1255.

[15] Patil, K., Mahima, V.G. and Ambika, L. (2007) Malignant Peripheral Nerve Sheath Tumors: An Elusive Diagnosis. Indian Journal of Dental Research, 18, 19-22. https://doi.org/10.4103/0970-9290.30917

[16] Lee, J.H., Lee, H.K., Choi, C.G., Suh, D.C., Lee, K.S. and Khang, S.K. (2001) Malignant Peripheral Nerve Sheath Tumor in Parapharyngeal Space: Tumor Spread through the Eustachian Tube. American Journal of Neuroradiology, 22, 748-750.

[17] Grobmyer, S., Reith, J.D., Shahlaee, A., Bush, C.H. and Hochwald, S.N. (2008) Malignant Peripheral Nerve Sheath Tumor: Molecular Pathogenesis and Current Management Considerations. Journal of Surgical Oncology, 97, 340-349. https://doi.org/10.1002/jso.20971

[18] Anghileri, M. and Miceli, R. (2006) Malignant Peripheral Nerve Sheath Tumor: Prognostic Factors and Survival in a Series of Patients Treated at a Single Institution. Cancer, 107, 1065-1074. https://doi.org/10.1002/cncr.22098

[19] Porter, D.E., Prasad, V., Foster, L., Dall, G.F., Birch, R. and Grimer, R.J. (2009) Survival in Malignant Peripheral Nerve Sheath Tumours: A Comparison between Sporadic and Neurofibromatosis Type 1-Associated Tumours. Sarcoma, 2009, Article ID: 756395 .

[20] Cunha, K.S.G., Caruso, A.C., de Faria, P.A.S., da Silva, L.E., Pires, A.R.C., Geller, M., Lopes, V.S. and de Moura-Neto, R.S. (2012) Malignant Peripheral Nerve Sheath Tumors: Clinico-Pathological Aspect, Expression of P53 and Survival. Clinics, 67, 963-968. https://doi.org/10.6061/clinics/2012(08)18

[21] Kosmas, C., Tsakonas, G., Evgenidi, K., Gassiamis, A., Savva, L., Mylonakis, N. and Karabelis, A. (2009) Malignant Peripheral Nerve Sheath Tumor in Neurobifromatosis Type-1: Two Case Reports. Cases Journal, 2, 7612.

https://doi.org/10.4076/1757-1626-2-7612 